\title{
Benign splenosis mimicking peritoneal seeding in a bladder cancer patient: a case report
}

\author{
Stefania Rizzo*1, Lorenzo Monfardini' ${ }^{1}$, Maddalena Belmonte ${ }^{2}$, \\ Bernardo Rocco ${ }^{3}$ and Massimo Bellomi ${ }^{1,2}$
}

Address: ${ }^{1}$ Department of Radiology, European Institute of Oncology, via Ripamonti 435, Milan, 20141, Italy, ${ }^{2}$ School of M 'icine, Unive) oity of Milan, via Festa del Perdono 7, Milan, 20122, Italy and '3 Department of Urology, European Institute of Oncology, Via Rivan, 20141, Italy

Email: Stefania Rizzo* - stefania.rizzo@ieo.it; Lorenzo Monfardini - lorenzo.monfardini@ieo.it; Maddalena Belmonte - maddalena.belmonte@ieo.it; Bernardo Rocco - bernardo.rocco@ieo.it; Massimo Bello ni - s simo.bellomi@ieo.it

* Corresponding author

Published: 9 December 2009

Cases Journal 2009, 2:9294 doi:10.1186/1757-1626-2-9294

This article is available from: http://www.casesjournal.com/content/2/I/9294

(c) 2009 Rizzo et al; licensee BioMed Central Ltd.

This is an Open Access article distributed under the terms of the Creative Commons Attributirn Ll,ense (http://creativecommons.org/licenses/by/2.0), which permits unrestricted use, distribution, and reproduction in any medium, provided the or iginal work is properly cited.
Abstract

Introduction: Splenosis is a post-tr-ma matot asplantation and proliferation of splenic tissue in ectopic sites. These implants $\mathrm{m}$ ?, nimic ma, nancy in healthy patients or peritoneal metastases in cancer patients. When a prev ous tory of splenic injury is known, the finding of soft tissue nodules in many thoracic anc'abciominal cations might raise the suspicion of the benign condition of splenosis, in order to av id unnecessary surgery or chemotherapy.

Case presentation: A 56 , n man with history of persistent hematuria from bladder cancer was referred to ou ctitution for suspected peritoneal carcinosis. For staging purposes he underwent abdomina $\mathrm{CS}_{\mathrm{in}}$ ited tomography and ultrasound. The integration of patient's history and imaging to ts lec to the diagnosis of peritoneal splenosis. The patient therefore underwent regular $T$. S $U$ athral Resection of Bladder for the known malignancy; while no treatment was necessary fo, Jenosis. Two years follow-up was negative for metastases.

C nc ion: Splenosis is a benign condition after traumatic splenectomy which should be taken nto acc $t z$ in the differential diagnosis with peritoneal seeding of malignancy because its appearance may resemble malignancy.

\section{Intre Iction}

Trauma, $1 \mathrm{c}$ disruption of the splenic capsule causes fragments of splenic tissue to be seeded throughout the peritoneal cavity [1]. It has been demonstrated that splenic implants can survive and grow when transplanted to ectopic sites, such as the splenic fossa, any site of the peri- toneal cavity, gastro-intestinal tract, liver, kidney, thorax, subcutaneous tissues and even in the head [2].

Since these implants may mimic malignancy or peritoneal metastases, in cancer patients with previous history of splenic injury, the finding of soft tissue nodules in the 
abovementioned locations might raise the suspicion of splenosis in order to avoid unnecessary surgery or chemotherapy.

This report describes the case of a bladder cancer patient with peritoneal splenosis mimicking metastases.

\section{Case presentation}

A 56-year-old white man native from Italy, was admitted to our Institution for a diagnosis of bladder cancer and a previous CT scan suggesting peritoneal carcinosis. His history started about 4 months in advance for the occurrence of hematuria. His previous personal history was unremarkable, other than a car accident about 20 years in advance, which requested a long hospital staying and a splenectomy.

At our hospital he underwent a Trans-Urethral Resection of Bladder (TURB) which led to the diagnosis of spinocellular carcinoma of the bladder. Usual patient's blood tests, including complete blood cell count and tumour markers, were unremarkable.

The patient underwent a second computed tomography (CT) scan for staging purposes, showing the presence of many peritoneal hypodense masses, with heterogeneous contrast-enhancement during the arterial phase and almost isodense to the hepatic parenchyma during the portal and equilibrium phases (Figure 1).

The spleen was not visualized on the CT sonn and 'enosis was thereafter suspected as a possib e diagnosis and a second-look ultrasound (US) exam c firmed the pres-

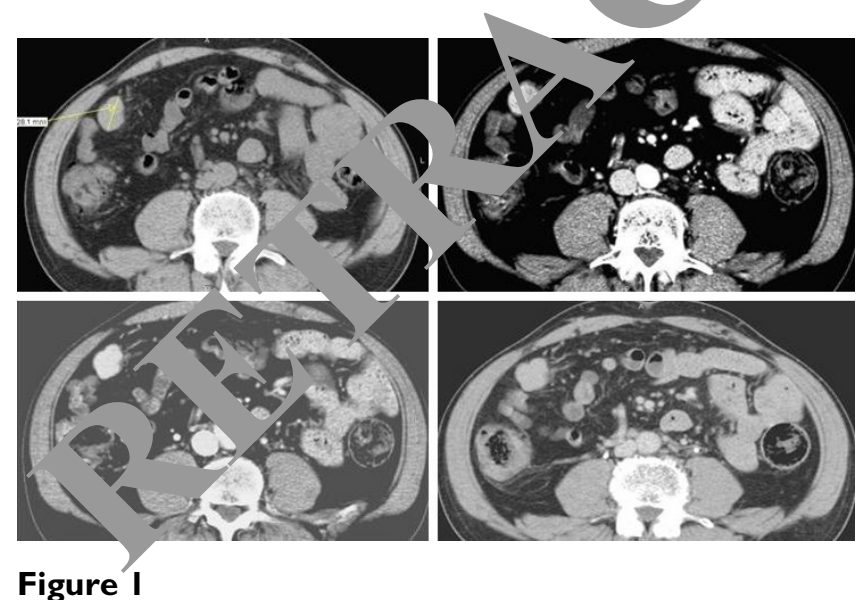

Figure I

Abdominal CT scan of a splenic nodule before and after contrast medium injection. This $28 \mathrm{~mm}$ peritoneal nodule located in the right paracolic space showed slight hypodensity during the pre-contrast phase (a), inhomogeneous contrast-enhancement during the arterial phase (b), slightly iso-hypodenity during the portal (c) and equilibrium phase (d). ence of peritoneal solid splenic-like masses on the sites indicated by CT (Figure 2).

Diagnosis of post-splenectomy splenosis was confirmed and the patient underwent usual TURV as treatment for bladder cancer, with no chemotherapy, or further treatment for splenosis.

\section{Discussion}

Splenosis is a relatively common fin ing in clit, cally silent patients, frequently misdiagn sea caus of the lack of symptoms. According to $N$ ormand $e_{\text {, it involves }}$ $16 \%$ to $67 \%$ of patients with pa splenic) trauma and/or past splenectomy [3]. On th oth hand, Khosravi et al found only five cases of $\mathrm{rr}^{\mathrm{r}} \mathrm{en}$ in a retrospective review of a 29-year period of t patholy / registry [1], while Lin et al identified eight atic with splenosis in a period of 8 years [2].

Based on the lo on or the splenic nodules, differential diagnose to be con yered may be endometriosis, in presence of $\mathrm{p}$ case of per toneal seeding [5], renal cancer [6] in case of romal impla ass, abdominal lymphomas in case of retropen neal locations mimicking lymph nodes [7], hepatic den mas in case of intra-hepatic implants [8] and peritoi. 1 metastases.

Thus, patient's history is substantial; secondarily imaging modalities to consider are: CT, US, magnetic resonance (MR), scintigraphy.

If the splenic implant is intra-hepatic, CT imaging may show hypodense masses with strong enhancement at the early phase and pooling enhancement at delayed phase [9]. Grande et al described multiple well-demarcated nod-

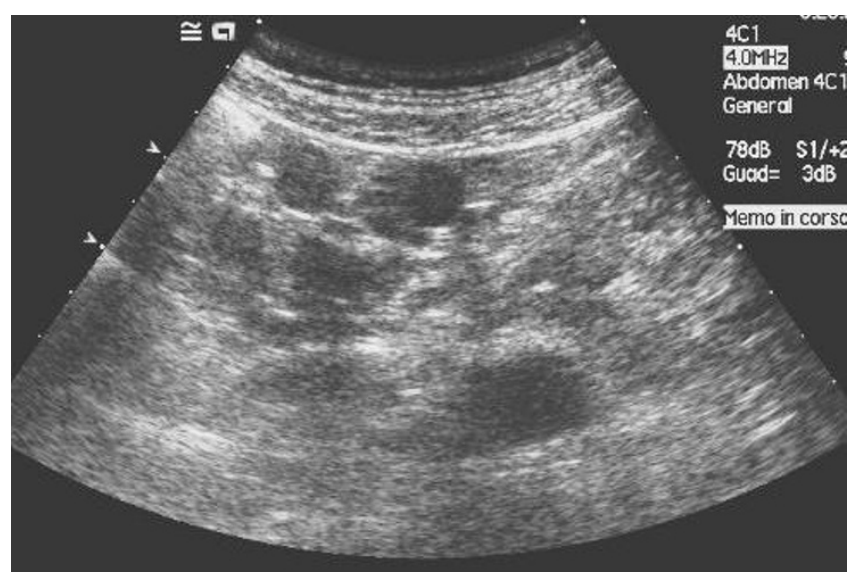

Figure 2

Second-look ultrasound of the nodule in figure I. US shows homogeneous splenic-like echogenicity of the nodule in the paracolic space, thus confirming its splenic origin. 
ules without calcifications, slightly hypodense compared to the liver at non-contrast CT, showing as lobular or oval well-circumscribed structures, hyperdense in the arterial phase and isodense in the portal phase after contrast administration [10]. Accordingly, Imbriaco described a well-demarcated intra-hepatic $3 \mathrm{~cm}$ mass, hypodense compared with the surrounding liver parenchyma at unenhanced CT, heterogeneously enhanced in the arterial phase, becoming hypodense during the portal end equilibrium phases [5].

In our case study, according to previous studies, CT scan showed multiple hypodense peritoneal masses with a maximum diameter of $15-20 \mathrm{~mm}$, which characteristically had slightly hypodensity in the pre-contrast phase, inhomogeneous contrast-enhancement during the arterial phase and iso - slightly hypodensity to the liver parenchyma during the portal and equilibrium phase.

Ultrasound may incidentally show hypoechoic masses on the surface of solid abdominal organs, such as the liver [9] or the kidney [5]. It may also show multiple solid masses with a smooth round or ovoid shape, with a homogeneous echo-texture and a hyperehoic peripheral rim, without specific arterial or venous Doppler signals [10]. In our case, a second-look ultrasound, confirmed the presence of multiple solid nodules with homogeneous splenic iike echogenicity in the splenic fossa and in the peritont

MRI may be considered as an alternative mo a for the identification of splenosis, in case of unce tainty diagnosis with other exams. Splenic im lants have been described as hypointense on T1-wei hted images and hyperintense on T2-wieghted images, $t$-for/similar to normal splenic tissue $[11,12]$.

Nowadays scintigraphy of ormed with heat-demaged 99Tc-labelled red bloo ell is still considered the more sensitive and specif. ima. $\mathrm{g}$ modality for the diagnosis of splenosis $[10,2,4]$, beil, sensitive even in early splenosis, in cases wher lenic tissue is minimally present, in functional hyposple $1 \mathrm{sm}$ and in case of poor splenic uptake. $\pi$ i cau se splenic tissue takes up more than $90 \%$ f dam d red blood cells [15].

On of is diagnosed with anyone of the previous moda $\circ$, no more treatment is usually required unless the patient is symptomatic.

\section{Conclusion}

In conclusion this report describes a case of a bladder cancer patient with peritoneal lesions related to personal history of post-trauma splenectomy (splenosis). Since this benign condition may mimic metastases, it should be kept in mind in managing cancer patients with personal history of post-traumatic splenectomy, in order to avoid unnecessary surgery or chemotherapy.

\section{Consent}

Written informed consent was obtained from the patient for publication of this case report. A copy of the written informed consent is available for review by the cditor-inChief of this journal.

\section{Competing interests}

The authors declare that they have no con ting nterests.

\section{Authors' contributions}

BR analyzed and interpreted e $\mathrm{p}$. nt $\mathrm{d}$ ata regarding the oncological disease and the r ested treatment. SR performed the radiologi exams cions (CT and ultrasound) of the abdon en a was the major contributor in writing the many ipt. $\mathrm{LM}$, MB contributed to the literature search $\mathrm{AB}$ s inervised the entire work. All authors read and appro, the rinal manuscript.

\section{Referenc.}

I. Khosrav IR, Yargulies DR, Alsabeh R, Nissen N, Phillips EH, Morgenstern :Consider the diagnosis of splenosis for soft tissue nasses I.nng after any splenic injury. Am Surg 2004, 70:967-970.

2. WC, Lee RC, Chiang JH, Wei CJ, Chu LS, Liu RS, Chang CY: MR f t tures of abdominal splenosis. AJR Am J Roentgenol 2003, I, 0:493-496.

3. Normand JP, Rioux M, Dumont M, Bouchard G, Letourneau L: Thoracic splenosis after blunt trauma: frequency and imaging findings. AJR Am J Roentgenol 1993, 161:739-741.

4. Zitzer P, Pansky M, Maymon R, Langer R, Bukovsky I, Golan A: Pelvic splenosis mimicking endometriosis, causing low abdominal mass and pain. Hum Reprod 1998, 13:1683-1685.

5. Imbriaco M, Camera L, Manciuria A, Salvatore M: A case of multiple intra-abdominal splenosis with computed tomography and magnetic resonance imaging correlative findings. World J Gastroenterol 2008, I 4: 1453-1455.

6. Kiser JW, Fagien M, Clore FF: Splenosis mimicking a left renal mass. AJR 1996, 167:1508-1509.

7. Schenkein DP, Ahmed E: Case records of the Massachusetts General Hospital. Weekly clinic-pathological exercises: Case 29-1995 A 65-year-old man with mediastinal Hodgkin's disease and a pelvic mass. N Engl J Med 1995, 333:784-79I.

8. Gruen DR, Gollub MJ: Intrahepatic splenosis mimicking hepatic adenoma. AJR Am J Roentgenol 1997, 168:725-726.

9. Nakajima T, Fujiwara A, Yamaguchi M, Makiyama A, Wakae T, Fujita K, Yoshikawa K, Shiomi T, Ohishi T, Nakashima T, Konishi E: Intrahepatic splenosis with severe iron deposition presenting with atypical magnetic resonance images. Intern Med 2008, 47:743-746.

10. Grande M, Lapecorella M, lanora AA, Longo S, Rubini G: Intrahepatic and widely distributed intraabdominal splenosis: multidetector CT, US and scintigraphic findings. Intern Emerg Med 2008, 3:265-267.

II. De Vuysere S, Van Steenbergen W, Aerts R, Van Hauwaert H, Van Beckevoort D, Van Hoe L: Intrahepatic splenosis: imaging features. Abdom Imaging 2000, 25:187-189.

12. Izzo L, Caputo M, Galati G: Intrahepatic accessory spleen: imaging features. Liver Int 2004:216-217.

13. Stewart CA, Sakimura IT, Siegel ME: Scintigraphic demonstration of splenosis. Clin Nucl Med 1986, II:161-164.

14. Zwas ST, Samra D, Samra Y, Sibber GR: Scintigraphic assessment of ectopic splenic tissue localization and function following splenectomy for trauma. Eur J Nucl Med 1986, I 2: I25-I29.

15. Fremont RD, Rice TW: Splenosis: a review. South Med J 2007, 100:589-593. 